\title{
Editorial: New Insights on Neuron and Astrocyte Function From Cutting-Edge Optical Techniques
}

\author{
Srdjan D. Antic ${ }^{1 *}$, Bradley James Baker ${ }^{2 \star}$ and Marco Canepari ${ }^{3,4,5 *}$ \\ ${ }^{1}$ Department of Neuroscience, Institute for Systems Genomics, Stem Cell Institute, UConn Health, Farmington, CT, \\ United States, ${ }^{2}$ The Center for Functional Connectomics, Korea Institute of Science and Technology, Seoul, South Korea, \\ ${ }^{3}$ Division of Bio-Medical Science and Technology, KIST School, Korea University of Science and Technology (UST), Seoul, \\ South Korea, ${ }^{4}$ Laboratories of Excellence, Ion Channel Science and Therapeutics, Valbonne, France, ${ }^{5}$ Institut National de la \\ Santé et Recherche Médicale, Paris, France
}

Keywords: optogenetic, voltage imaging, calcium imaging, GCaMP, voltage-sensitive dye

\section{Editorial on the Research Topic}

\section{New Insights on Neuron and Astrocyte Function From Cutting-Edge Optical Techniques}

One of the most coveted goals of science is to elucidate the cellular bases of complex brain functions. How does a piece of biological tissue produce accurate cognitive insights, or succumbs to a devastating neurological disease? Neurons and astrocytes are the most numerous cells in the brain, comprising $>95 \%$ of the brain parenchyma, and are responsible for all aspects of rapid information processing in the mammalian brain. Monitoring of physiological changes at the individual cell level, as well as in cell populations, is essential (Popovic M. et al., 2015; Nakajima et al., 2016). Early explorations of cellular physiology entailed metal (or glass and metal) electrodes. However, due to their highly invasive and tissue-harming nature, all electrodes, no matter how small, are limited experimental tools. While understanding brain circuits ultimately relies on the analyses of multiple and well-identified cells and subcellular compartments, the required information at high spatial and temporal resolution is not achievable using only electrode techniques for several reasons. First, the necessity for simultaneous measurements from ensembles of cells seems to be the most agreed upon aspect of the modern neuroscience (Barbera et al., 2016; Cai et al., 2016; Ji et al., 2016). Unfortunately, electrode arrays are limited in the number of cells they can monitor simultaneously. Second, the other reason for choosing optical imaging technologies over standard electrode recordings is that this is the only available approach to explore in detail thin processes of neurons and astrocytes, i.e., the essential cellular compartments underlying brain function (Larkum et al., 2018; Sheffield and Dombeck, 2019). In particular, in thin dendritic branches of neurons and astrocytes, optical measurements perform better than electrode recordings, in terms of calcium activity (Schiller et al., 1995; Kampa et al., 2006), membrane potential transients (Zhou et al., 2008), and also for recording ion currents (Jaafari et al., 2014; Jaafari and Canepari, 2016; Ait Ouares et al., 2019). In summary, the places where electrodes cannot go, optical measurements are taking over (Popovic M. A. et al., 2015; Bindocci et al., 2017; Gordleeva et al., 2019).

In the last decades, neuroscience research has been driven by the development of novel technologies interfacing scientists with molecules, cells, neuronal tissues, or the entire brain. Certainly, optical techniques have played a major role in this progress opening the gate to unprecedented information on living neurons and astrocytes. Innovative illumination and light recording strategies (Müllenbroich et al.; Ronzitti et al.; Battefeld et al.; Miyazaki et al.) advanced our knowledge on signaling in submicron structures (Francavilla et al.; Kuhn and Roome; Miyazaki et al.) while permitting the investigations in semi-intact tissues (Tominaga et al.; Kajiwara et al.; Quicke et al.). In parallel, the new emerging field of optogenetics allowed the selected expression 
of light-activated proteins that can be used for precisely targeted photostimulation (Baker and Flannery). Other optical strategies that recently progressed include combined $\mathrm{Ca}^{2+}$ and $\mathrm{Na}^{+}$ measurements (Miyazaki et al.), membrane potential recordings from individual cells (Francavilla et al.; Kuhn and Roome; Quicke et al.), or cell populations (Tominaga et al.; Kajiwara et al.; Li et al.), and chemical stimulation of targeted receptors using caged compounds (Palma-Cerda et al.).

\section{STUDIES USING ORGANIC INDICATORS}

In the early days of functional optical imaging, organic dyes were used for studying membrane potential changes in excitable cells and were dubbed voltage-sensitive dyes (Davila et al., 1973; Cohen et al., 1978). Later on, compounds were synthesized for measuring calcium or sodium transients in the cytosol (Brown et al., 1975; Grynkiewicz et al., 1985). Tominaga et al. have developed a voltage-sensitive dye imaging method for measuring compound synaptic potentials (population signals) from the entire hippocampus CA1 area for extended periods of time (experiments lasting up to $12 \mathrm{~h}$ ). Their results shed light on a rarely discussed spatial aspect of long term potentiation (LTP) induction (e.g., magnitude of LTP increases with distance from the stimulation site) (Tominaga et al.). Using the same experimental approach, based on extracellular labeling of brain slices with voltage-sensitive dye and state-of-the-art wide-field single-photon voltage imaging at 2,000 frames per s, Kajiwara et al. showed that the efficacy of propagation of electrical activity between two critical brain regions, from perirhinal cortex to entorhinal cortex, is strongly dependent on membrane excitability and is malleable by synaptic plasticity (Kajiwara et al.). Miyazaki et al. have developed a robust method for simultaneous imaging of $\left[\mathrm{Ca}^{+}\right]_{\mathrm{i}}$ and $\left[\mathrm{Na}^{+}\right]_{\mathrm{i}}$ changes in neurons at high speed and high spatial resolution. Via whole-cell electrode attached to the cell body, the authors co-inject two different pairs of spectrally orthogonal $\mathrm{Ca}^{2+}$ and $\mathrm{Na}^{+}$indicators and excite the two dyes by illuminating at two wavelengths either using LEDs or laser spots. This method allowed them to monitor synaptically evoked $\mathrm{Ca}^{2+}$ and $\mathrm{Na}^{+}$transients in individual dendritic spines (Miyazaki et al.). The review article by Dong et al. describes several techniques for studying gap junctions in the central nervous system. Fluorescent compounds and dyes are at the heart of several experimental approaches designed to probe the existence, strength, or nature of gap junction coupling: injection of small tracer molecules, scrape loading/dye transfer method, and recovery after photobleaching method (e.g., membranepermeable fluorescein-AM). The review article by Kuhn and Roome, describes experimental design for performing dendritic voltage imaging in awake behaving animals. The authors also provide a very detailed account of the physical chemistry and quantum mechanics of charge-shift voltage-sensitive dyes with a special emphasis on two dyes from the Fromherz group (ANNINE-6 and ANNINE-6+). Physical chemistry and quantum mechanics of the organic dyes explains certain practical issues of working with fluorescent dyes, such as signal-tonoise ratio, phototoxicity, shape of the emission spectrum, dye flipping from one membrane leaf to another, etc. (Kuhn and Roome).

\section{STUDIES USING GENETICALLY ENCODED INDICATORS}

Genetically encoded optical sensors of cell activity have become very popular in recent years, because they can be targeted to specific cell types. This is especially important in studies of the brain circuitry, because individual brain regions always include a multitude of different cell types, each type bestowed with a specific set of afferents and efferents, as well as rules for synaptic integration and patterned action potential firing. Francavilla et al. used viral transfection to label hippocampal GABAergic interneurons with the calcium indicator GCaMP6f. Two photon imaging of dendritic calcium transients (CaTs) was carried out in head-restrained mice running on a circular treadmill. Data showed that $\mathrm{Ca}^{2+}$ signals had larger amplitude and could invade the entire dendritic tree during locomotion, while during immobility, the signal amplitude was significantly lower in both soma and dendrites, and a significant fraction of dendrites showed spatially restricted CaTs, which were not seen in the soma. The authors proposed that strong dendrite-soma coupling, associated with animal locomotion, may facilitate the spike-timing-dependent or Hebbian forms of synaptic plasticity in interneurons. In contrast, during animal quiet state, this type of activity can be reduced or even replaced by local dendritic $\mathrm{Ca}^{2+}$ signaling, which may facilitate the anti-Hebbian plasticity mechanisms (Francavilla et al.). Another group working with GCaMP used the transgenic animal Thy1-GCaMP6f, expressing GCaMP6f in cortical pyramidal neurons (Li et al.). These authors showed that they can monitor sharp waves (SWs), carbacholinduced theta oscillations, and interictal-like spikes in brain slices. Furthermore, the authors claim that population GCaMP6f signals are fast enough for monitoring theta and beta oscillations $(<25 \mathrm{~Hz})$, which is an advancement for $\mathrm{Ca}^{2+}$ imaging. Quicke et al. also use a transgenic animal approach for delivery of a genetically encoded indicator to neurons. However, instead of GCaMP which is a calcium indicator, they use voltage indicator (VSFP Butterfly 1.2) to monitor membrane potential changes (Quicke et al.). Unlike the cytosolic molecule GCaMP, voltage indicators are membrane proteins (Kannan et al.). Their expression in brain tissue produces fluorescently labeled neuropil made of densely intermingled dendrites and axons, hence cellular resolution is lost. To achieve single cell resolution in voltage imaging, Quicke et al. combine recently developed sparse transgenic expression strategy (Song et al., 2017) and patch clamp recordings to demonstrate optical recordings of action potentials in individual neurons (Quicke et al.). Unlike GCaMP, genetically encoded voltage indicators have poor signal to noise ratio (Antic et al., 2016; Storace et al., 2016). But the signal-quality gap between GCaMP and GEVI is becoming narrower each year (Adam et al., 2019; Qian et al., 2019). A set of the state-of-the-art strategies for improving GEVI properties (sensitivity, brightness, SNR, fast kinetics, etc.) is described in an excellent article by Kannan et al., together with the overview of 
the pallet of currently available GEVIs (Kannan et al.). Finally, Storace et al. illustrate how membrane potential imaging using GEVIs (e.g., Arclight) can be utilized to decode the activity of the mammalian olfactory bulb, a goal that has been elusive for decades using older experimental approaches. Here, the authors used a clever strategy to simultaneously monitor input and output of a brain region in awake animal responding to controlled odor presentations. Afferent inputs to the olfactory glomeruli were assessed by calcium imaging from axon terminals of olfactory receptor cells (impinging onto the tufts of the mitral cells in the olfactory bulb glomerulus). Olfactory bulb outputs, on the other hand, were monitored by GEVI labeling of the mitral cells, whose axons project from the olfactory bulb into other brain regions. Simultaneous calcium and voltage imaging revealed that the output activity maps and the output signal size are much less concentration dependent than are the input maps and signals (Storace et al.). These results contribute immensely to our current understanding of the cellular mechanism for concentration invariance of odor recognition. The Storace et al. study is an excellent example of how applications of advanced imaging technologies (dual anatomical and genetic targeting leading to simultaneous calcium voltage imaging in an awake behaving animal) will impact systems neuroscience in the future.

\section{ADVANCES OF OPTICAL TECHNOLOGIES}

The ability of functional imaging to address fundamental questions depends on novel cutting-edge technologies and instrumentation. Battefeld et al. describe a versatile and open-source rapid LED switching system for one-photon imaging and photo-activation, that can be used either for quantitative ratiometric $\mathrm{Ca}^{2+}$ imaging or to combine $\mathrm{Ca}^{2+}$ imaging with optogenetic stimulation. Miyazaki et al. present an upgraded version of an imaging system, based on high speed multiplexing of light LEDs, to combine $\mathrm{Na}^{+}$and $\mathrm{Ca}^{2+}$ imaging, a powerful approach to advance our knowledge on neuronal excitability and synaptic transmission. Ronzitti et al. review strategies for light shaping in three dimensions, including SLM-based multiplexing illumination, temporal focusing and other approaches. These rapidly developing illumination technologies open the gate to all-optical manipulation of neural circuits involving simultaneous stimulation of welldefined targets and simultaneous functional imaging of the resulting activity. Among light shaping approaches, light-sheet microscopy represents one of the most promising strategies. Using this technique, Müllenbroich et al. present an interesting improvement for imaging the transparent nervous system of zebra fish. In this application, Bessel beams are used to remove artifacts and dramatically improve sensitivity in $\mathrm{Ca}^{2+}$ imaging measurements (Müllenbroich et al.). Finally, a novel caged glutamate receptor antagonist is presented by Palma-Cerda et al. This compound combines the fast photolysis and hydrolytic stability of this family of photoactivatable molecules with fast-equilibrating competitive action on glutamate receptors, enabling deep investigation of postsynaptic functions at the molecular level (Palma-Cerda et al.).

\section{AUTHOR CONTRIBUTIONS}

All authors listed have made a substantial, direct and intellectual contribution to the work, and approved it for publication.

\section{FUNDING}

SA has been supported by National Institutes of Health (NIH) grants MH109091, NS099573, EB022903; and Cure Alzheimer's Fund. MC has been supported by the Agence Nationale de la Recherche through the grants ANR-14-CE17-0006 and ANR-11LABX-0015. BB has been supported by the National Institute of Neurological Disorders And Stroke of the National Institutes of Health under Award Number U01NS099691. The content is solely the responsibility of the authors and does not necessarily represent the official views of the National Institutes of Health. BB has also been funded by the Korea Institute of Science and Technology (KIST) grants 2E26190 and 2E26170.

\section{REFERENCES}

Adam, Y., Kim, J. J., Lou, S., Zhao, Y., Xie, M. E., Brinks, D., et al. (2019). Voltage imaging and optogenetics reveal behaviour-dependent changes in hippocampal dynamics. Nature 569, 413-417. doi: 10.1038/s41586-019-1166-7

Ait Ouares, K., Filipis, L., Tzilivaki, A., Poirazi, P., and Canepari, M. (2019). Two distinct sets of $\mathrm{Ca}^{2+}$ and $\mathrm{K}^{+}$channels are activated at different membrane potentials by the climbing fiber synaptic potential in purkinje neuron dendrites. J. Neurosci. 39, 1969-1981. doi: 10.1523/JNEUROSCI.2155-18.2018

Antic, S. D., Empson, R. M., and Knopfel, T. (2016). Voltage imaging to understand connections and functions of neuronal circuits. J. Neurophysiol. 116, 135-152. doi: 10.1152/jn.00226.2016

Barbera, G., Liang, B., Zhang, L., Gerfen, C. R., Culurciello, E., Chen, R., et al. (2016). Spatially compact neural clusters in the dorsal striatum encode locomotion relevant information. Neuron 92, 202-213. doi: 10.1016/j.neuron.2016.08.037

Bindocci, E., Savtchouk, I., Liaudet, N., Becker, D., Carriero, G., and Volterra, A. (2017). Three-dimensional $\mathrm{Ca}^{2+}$ imaging advances understanding of astrocyte biology. Science 356:eaai8185. doi: 10.1126/science.aai8185
Brown, J. E., Cohen, L. B., De Weer, P., Pinto, L. H., Ross, W. N., and Salzberg, B. M. (1975). Rapid changes in intracellular free calcium concentration. Detection by metallochromic indicator dyes in squid giant axon. Biophys. J. 15, 1155-1160. doi: 10.1016/S0006-3495(75)85891-7

Cai, D. J., Aharoni, D., Shuman, T., Shobe, J., Biane, J., Song, W., et al. (2016). A shared neural ensemble links distinct contextual memories encoded close in time. Nature 534, 115-118. doi: 10.1038/nature 17955

Cohen, L. B., Salzberg, B. M., and Grinvald, A. (1978). Optical methods for monitoring neuron activity. Annu.Rev. Neurosci. 1, 171-182. doi: 10.1146/annurev.ne.01.030178.001131

Davila, H. V., Salzberg, B. M., Cohen, L. B., and Waggoner, A. S. (1973). A large change in axon fluorescence that provides a promising method for measuring membrane potential. Nat. New Biol. 241, 159-160. doi: 10.1038/newbio241159a0

Gordleeva, S. Y., Ermolaeva, A. V., Kastalskiy, I. A., and Kazantsev, V. B. (2019). Astrocyte as spatiotemporal integrating detector of neuronal activity. Front. Physiol. 10:294. doi: 10.3389/fphys.2019. 00294 
Grynkiewicz, G., Poenie, M., and Tsien, R. Y. (1985). A new generation of $\mathrm{Ca}^{2+}$ indicators with greatly improved fluorescence properties. J. Biol. Chem. 260, 3440-3450.

Jaafari, N., and Canepari, M. (2016). Functional coupling of diverse voltage-gated $\mathrm{Ca}^{2+}$ channels underlies high fidelity of fast dendritic $\mathrm{Ca}^{2+}$ signals during burst firing. J. Physiol. 594, 967-983. doi: 10.1113/JP271830

Jaafari, N., De Waard, M., and Canepari, M. (2014). Imaging fast calcium currents beyond the limitations of electrode techniques. Biophys. J. 107, 1280-1288. doi: 10.1016/j.bpj.2014.07.059

Ji, N., Freeman, J., and Smith, S. L. (2016). Technologies for imaging neural activity in large volumes. Nat. Neurosci. 19, 1154-1164. doi: 10.1038/ nn. 4358

Kampa, B. M., Letzkus, J. J., and Stuart, G. J. (2006). Requirement of dendritic calcium spikes for induction of spike-timing-dependent synaptic plasticity. J. Physiol. 574, 283-290. doi: 10.1113/jphysiol.2006.111062

Larkum, M. E., Petro, L. S., Sachdev, R. N. S., and Muckli, L. (2018). A perspective on cortical layering and layer-spanning neuronal elements. Front. Neuroanat. 12:56. doi: 10.3389/fnana.2018.00056

Nakajima, R., Jung, A., Yoon, B. J., and Baker, B. J. (2016). Optogenetic monitoring of synaptic activity with genetically encoded voltage indicators. Front. Synaptic. Neurosci. 8:22. doi: 10.3389/fnsyn.2016.00022

Popovic, M., Vogt, K., Holthoff, K., Konnerth, A., Salzberg, B. M., Grinvald, A., et al. (2015). Imaging submillisecond membrane potential changes from individual regions of single axons, dendrites and spines. Adv. Exp. Med. Biol. 859, 57-101. doi: 10.1007/978-3-319-17641-3_3

Popovic, M. A., Carnevale, N., Rozsa, B., and Zecevic, D. (2015). Electrical behaviour of dendritic spines as revealed by voltage imaging. Nat. Commun. 6:8436. doi: $10.1038 /$ ncomms 9436

Qian, Y., Piatkevich, K. D., Mc Larney, B., Abdelfattah, A. S., Mehta, S., Murdock, M. H., et al. (2019). A genetically encoded near-infrared fluorescent calcium ion indicator. Nat. Methods 16, 171-174. doi: 10.1038/s41592-018 0294-6

Schiller, J., Helmchen, F., and Sakmann, B. (1995). Spatial profile of dendritic calcium transients evoked by action potentials in rat neocortical pyramidal neurones. J. Physiol. 487, 583-600. doi: 10.1113/jphysiol.1995.sp020902

Sheffield, M. E., and Dombeck, D. A. (2019). Dendritic mechanisms of hippocampal place field formation. Curr. Opin. Neurobiol. 54, 1-11. doi: 10.1016/j.conb.2018.07.004

Song, C., Do, Q. B., Antic, S. D., and Knöpfel, T. (2017). Transgenic strategies for sparse but strong expression of genetically encoded voltage and calcium indicators. Int. J. Mol. Sci. 18:E1461. doi: 10.3390/ijms18071461

Storace, D., Sepehri Rad, M., Kang, B., Cohen, L. B., Hughes, T., and Baker, B. J. (2016). Toward better genetically encoded sensors of membrane potential. Trends Neurosci. 39, 277-289. doi: 10.1016/j.tins.2016.02.005

Zhou, W. L., Yan, P., Wuskell, J. P., Loew, L. M., and Antic, S. D. (2008). Dynamics of action potential backpropagation in basal dendrites of prefrontal cortical pyramidal neurons. Eur. J. Neurosci. 27, 923-936. doi: $10.1111 /$ j.1460-9568.2008.06075.x

Conflict of Interest: The authors declare that the research was conducted in the absence of any commercial or financial relationships that could be construed as a potential conflict of interest.

Copyright (c) 2019 Antic, Baker and Canepari. This is an open-access article distributed under the terms of the Creative Commons Attribution License (CC BY). The use, distribution or reproduction in other forums is permitted, provided the original author(s) and the copyright owner(s) are credited and that the original publication in this journal is cited, in accordance with accepted academic practice. No use, distribution or reproduction is permitted which does not comply with these terms. 